\title{
CONSTANT SCALAR CURVATURE HYPERSURFACES WITH SECOND-ORDER UMBILICITY
}

\author{
ANTONIO GERVASIO COLARES \\ Departamento de Matemática, Universidade Federal do Ceará, Campus do Pici, 60455-760 Fortaleza-CE, \\ Brazil \\ e-mail:gcolares@mat.ufc.br \\ and FERNANDO ENRIQUE ECHAIZ-ESPINOZA \\ Instituto de Matemática, Universidade Federal de Alagoas, Campus A.C. Simões, 57455-760 Maceió-AL, \\ Brazil \\ e-mail:echaiz@pos.mat.ufal.br
}

(Received 22 April 2008; accepted 21 August 2008)

\begin{abstract}
We extend the concept of umbilicity to higher order umbilicity in Riemannian manifolds saying that an isometric immersion is $k$-umbilical when $A P_{k-1}(A)$ is a multiple of the identity, where $P_{k}(A)$ is the $k$ th Newton polynomial in the second fundamental form $A$ with $P_{0}(A)$ being the identity. Thus, for $k=1$, oneumbilical coincides with umbilical. We determine the principal curvatures of the twoumbilical isometric immersions in terms of the mean curvatures. We give a description of the two-umbilical isometric immersions in space forms which includes the product of spheres $S^{k}\left(\frac{1}{\sqrt{2}}\right) \times S^{k}\left(\frac{1}{\sqrt{2}}\right)$ embedded in the Euclidean sphere $S^{2 k+1}$ of radius 1 . We also introduce an operator $\phi_{k}$ which measures how an isometric immersion fails to be $k$-umbilical, giving in particular that $\phi_{1} \equiv 0$ if and only if the immersion is totally umbilical. We characterize the two-umbilical hypersurfaces of a space form as images of isometric immersions of Einstein manifolds.
\end{abstract}

2000 Mathematics Subject Classification. Primary 53C42, 53C40; Secondary 53B20, 53B25.

1. Introduction. Let $x: \mathbf{M}^{n} \rightarrow \overline{\mathbf{M}}^{n+1}, n \geq 2$, be an isometric immersion of a Riemannian manifold $\mathbf{M}^{n}$ in a Riemannian manifold $\overline{\mathbf{M}}^{n+1}$. We know that $x$ is totally umbilical if for each $p \in \mathbf{M}^{n}$ the second fundamental form $A_{p}: T_{p} \mathbf{M} \rightarrow T_{p} \mathbf{M}$ is a multiple of the identity on $T_{p} \mathbf{M}$. That is, if $\lambda_{1}(p), \ldots, \lambda_{n}(p)$ are the eigenvalues of $A_{p}$, then $S_{1}=\sum_{i=1}^{n} \lambda_{i}$ is constant and so

$$
A_{p}=\frac{S_{1}}{n} I
$$

$I$ the identity of $T_{p} \mathbf{M}$.

We extend the concept of umbilicity to higher order umbilicity, calling $k$-umbilicity, for $k=1, \ldots, n$. We say that an isometric immersion $x$ is $k$-umbilical (or has umbilicity of order $k$ ) if, at each point $p \in \mathbf{M}, A P_{k-1}$ is a multiple of the identity. Here, $P_{k}$ is the Newton polynomial in the second fundamental form $A$ on $T_{p} \mathbf{M}$ given, inductively, by 
$P_{0}=I, P_{k}=S_{k} I-P_{k-1} A$. If $A P_{k-1} \equiv 0$ we say that $x$ is $k$-totally geodesic. Here,

$$
S_{k}=\sum_{1 \leq i_{1}<i_{2}<\cdots<i_{k} \leq n} \lambda_{i_{1}} \lambda_{i_{2}} \ldots \lambda_{i_{k}} .
$$

The $k$ th mean curvature $H_{k}$ is given by

$$
H_{k}=\frac{1}{\left(\begin{array}{l}
n \\
k
\end{array}\right)} S_{k} .
$$

Thus, one-umbilical immersion is the known totally umbilical immersion. A $k$ umbilical isometric immersion in a Riemannian manifold satisfies (cf. Theorem 5.5): $A P_{k-1}(A)$ is a Codazzi tensor if and only if $S_{k}$ is constant (known for $k=1$ ). An interesting but different concept of $k$-umbilicity was introduced and developed in [2] and [7]. To study $k$-umbilicity we define an operator $\phi_{k}$ on tangent spaces of the immersion given by

$$
\phi_{k}(X)=\frac{k}{n} S_{k}(A) X-A P_{k-1}(A) X .
$$

We will prove that $\phi_{k}=0$ if and only if the immersion is $k$-umbilical (cf. Remark 5.7). For $k=1$ this was studied in [1].

An example of a two-umbilical embedding is given by $S^{k}\left(\frac{1}{\sqrt{2}}\right) \times S^{k}\left(\frac{1}{\sqrt{2}}\right) \rightarrow$ $S^{2 k+1}(1)$.

We prove that if $x$ is $k$-umbilical, then

$$
H_{k+1}=H_{1} H_{k}
$$

But there exist hypersurfaces satisfying the condition $H_{k+1}=H_{1} H_{k}$ that are not $k$-umbilical. For example, consider

$$
x: S O(3) \longrightarrow \quad S^{4}(r)
$$

letting

$$
g \longmapsto g\left(\begin{array}{ccc}
\frac{r \sqrt{2}}{2} & 0 & 0 \\
0 & \frac{-r \sqrt{2}}{2} & 0 \\
0 & 0 & 0
\end{array}\right) g^{-1}
$$

where $S^{4}(r)$ is the Euclidean sphere. We see that $\lambda_{1}=0, \lambda_{2}=\frac{\sqrt{3}}{2}$ and $\lambda_{3}=-\frac{\sqrt{3}}{2}$ and so $A P_{1}$ is not a multiple of the identity, hence $x$ is not two-umbilical. Clearly, $H_{3}=$ $H_{1} H_{2}$, because $S_{1}=0=S_{3}$. However, the condition $H_{1} H_{k}=H_{k+1}$ characterizes the $k$-umbilical isometric immersions whose principal curvatures never vanish (cf. Theorem 7.1).

We will show that for $n \geq 3$, if $x$ is $k$-umbilical in a space form $\mathbf{M}^{n+1}(c)$, then $S_{k}$ is constant. For $n=2$ this is not true because every immersion $x$ of $M^{3}(c)$ is twoumbilical, since the eigenvalues of $A P_{1}$ are equal to the Gaussian curvature of $x$. This should not be surprise, since it would happen with the concept of umbilicity extended to one-dimensional immersion in $M^{2}(c)$ : every curve would be umbilical. 
In this paper, we will study $k$-umbilical isometric immersions in Riemannian manifolds. We concentrate mainly on two-umbilical isometric immersions. First, we will prove the following theorem (cf. Theorem 8.1) on the determination of the principal curvatures of the two-umbilical isometric immersions in a Riemannian manifold:

Let $x: \mathbf{M}^{n} \longrightarrow \overline{\mathbf{M}}^{n+1}(n \geq 3)$ be any two-umbilical isometric immersion.

(a) If its principal curvatures are distinct, then they are given by

$$
\lambda_{i_{1}}=\cdots=\lambda_{i_{r}}=((n-(r+1)) /(n-2 r)) S_{1}
$$

and

$$
\lambda_{i_{r+1}}=\cdots=\lambda_{i_{n}}=-((r-1) /(n-2 r)) S_{1},
$$

where $r \in\left\{0,1,2, \ldots,\left[\left[\frac{n}{2}\right]\right]^{1}\right\}$ or $r \in\left\{0,1,2, \ldots, \frac{n}{2}-1\right\}$, according to whether $n$ is odd or even, respectively.

(b) If its principal curvatures are equal, then $n$ is even and its principal curvatures are given by

$$
\lambda_{i_{1}}=\cdots=\lambda_{i_{\frac{n}{2}}}=\sqrt{2 / n} \sqrt{-S_{2}}
$$

and

$$
\lambda_{i_{\frac{n}{2}+1}}=\cdots=\lambda_{i_{n}}=-\sqrt{2 / n} \sqrt{-S_{2}}
$$

We give a description of an infinite family of two-umbilical hypersurfaces in the sphere $S^{n+1}(1)$ (cf. Theorem 9.3):

There exists a countably infinite family of two-umbilical hypersurfaces in the Euclidean sphere $S^{n+1}(1)$ : for any $n \geq 4$ and for every $m \in\{2, \ldots, n-2\}$, the Clifford's hypersurface

$$
S^{n-m}(r) \times S^{m}\left(\sqrt{1-r^{2}}\right) \hookrightarrow S^{n+1}(1) \quad \text { is two-umbilical if and only if } \quad r=\sqrt{\frac{n-m-1}{n-2}} .
$$

We will see that for $r=\sqrt{\frac{n-m-1}{n-2}}$ the hypersurface $S^{n-m}(r) \times S^{m}\left(\sqrt{1-r^{2}}\right) \hookrightarrow$ $S^{n+1}(1)$ with the metric induced from $S^{n+1}(1)$ is an Einstein manifold. In fact, $S_{2}=-\frac{n}{2}$ and $\operatorname{Ric}(X, Y)=\left(n-1+\frac{2 S_{2}}{n}\right)<X, Y>$, when $r=\sqrt{\frac{n-m-1}{n-2}}$. That is, for $r=\sqrt{\frac{n-m-1}{n-2}}$, the two-umbilical Clifford hypersurfaces are examples of Einstein manifolds which admit isometric immersion in $S^{n+1}(1)$.

In [8], Fialkow gave a classification of the Einstein hypersurfaces in space forms. By using our methods we reprove part of Theorem 7.1 of [8]:

Every connected Einstein hypersurface in a space form has at most two distinct principal curvatures ( $c f$. Theorem 4.1 ).

The classical reference [4] has characterizations of Einstein manifolds under many distinct aspects.

Here, we will prove a characterization of Einstein hypersurfaces in space forms in terms of the second-order umbilicity (cf. Theorem 8.3):

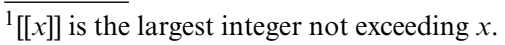


Let $\mathbf{M}^{n}$ be a connected Riemannian manifold and $x: \mathbf{M}^{n} \longrightarrow \overline{\mathbf{M}}^{n+1}(c), n \geq 3$, be an isometric immersion. Then

$\mathbf{M}^{n}$ is Einstein if and only if $x$ is two-umbilical.

Moreover, in this case Ric $(X, Y)=\left(c(n-1)+\frac{2 S_{2}}{n}\right)<X, Y>$, with $S_{2}$ constant

As a consequence, this yields $\phi_{2}$ as a measure of how much $\mathbf{M}^{n}$ fails to be an Einstein hypersurface (cf. Remark 8.4).

Our methods offer the possibility of studying $k$-umbilical isometric immersions in more general ambient spaces, as in Theorem 8.1.

By using Theorem 8.1, the paper ends with a description of the two-umbilical hypersurfaces in a space form (cf. Theorem 9.6):

Let $\mathbf{M}^{n}$ be a two-umbilical hypersurface in $\overline{\mathbf{M}}^{n+1}(c), n>2$. Then

(a) $\mathbf{M}$ is two-totally geodesic or

(b) $\mathbf{M}$ is one-umbilical or

(c) if $c>0$, then $\mathbf{M}$ is locally a standard product embedding of

$$
S^{n-m}(r) \times S^{m}\left(\sqrt{1-r^{2}}\right) \hookrightarrow S^{n+1}(1),
$$

where $r=\sqrt{\frac{n-m-1}{n-2}}$. In particular, when the embedding is minimal we have

$$
S^{k}\left(\frac{1}{\sqrt{2}}\right) \times S^{k}\left(\frac{1}{\sqrt{2}}\right) \hookrightarrow S^{2 k+1}(1),
$$

where $n=2 k$;

(d) if $c<0$, then $\mathbf{M}$ is geodesic hyperspheres, horospheres, totally geodesic hyperplanes and their equidistant hypersurfaces, tubes around totally geodesic subspaces of dimension at least one (in another words, it is locally a standard product embedding of $\left.S^{k} \times \mathbb{\boxplus}^{n-k}\right)$;

(e) if $c=0$, then $\mathbf{M}$ is locally hyperspheres, hyperplanes or a standard product embedding given by $S^{k} \times \mathbb{R}^{n-k}$.

2. Preliminaries. In this section, we fix notation and recall basic concepts that will be extended to self-adjoint operators in the next section.

DeFinition 2.1. Given any integer $k$, the function $S_{k}: \mathbb{R}^{n} \longrightarrow \mathbb{R}$ given by

$$
S_{k}\left(x_{1}, x_{2}, \ldots, x_{n}\right):= \begin{cases}1, & k=0, \\ \sum_{1 \leq i_{1}<i_{2}<\cdots<i_{k} \leq n} x_{i_{1}} x_{i_{2}} \ldots x_{i_{k}}, & \forall k \in\{1,2, \ldots, n\}, \\ 0, & \forall k \in \mathbb{Z} \backslash\{0, \cdots, n\}\end{cases}
$$

will be called an elementary $k$-symmetric polynomial.

DEFINITION 2.2. Given any integer $k$, let $S_{k}$ be the $k$-symmetric polynomial as given in Definition 2.1. We define the $j$ th partial derivative of $S_{k}$ by the following recurrence 
relations:

$$
\begin{aligned}
\frac{\partial}{\partial x_{j}} S_{0}\left(x_{1}, \ldots, x_{n}\right) & =0 \\
\frac{\partial}{\partial x_{j}} S_{1}\left(x_{1}, \ldots, x_{n}\right) & =1 \\
\frac{\partial}{\partial x_{j}} S_{r}\left(x_{1}, \ldots, x_{n}\right) & =S_{r-1}\left(x_{1}, \ldots, x_{n}\right)-x_{j} \frac{\partial}{\partial x_{j}} S_{r-1}\left(x_{1}, \ldots, x_{n}\right), \quad \forall r \geq 2 .
\end{aligned}
$$

From these relations, we can show by induction that

$$
\frac{\partial}{\partial x_{j}} S_{r+1}\left(x_{1}, \ldots, x_{n}\right)=\sum_{i=0}^{r}(-1)^{i} S_{r-i}\left(x_{1}, \ldots, x_{n}\right)\left(x_{j}\right)^{i} .
$$

Proposition 2.3. Given any integer $k$, let $S_{k}: \mathbb{R}^{n} \longrightarrow \mathbb{R}$ be the elementary $k$-symmetric polynomial. Then

$$
S_{k}\left(x_{1}, \ldots, \widehat{x}_{j}, \ldots, x_{n}\right)=\sum_{i=0}^{k}(-1)^{i} S_{k-i}\left(x_{1}, \ldots, x_{n}\right)\left(x_{j}\right)^{i},
$$

where $\widehat{x}_{j}$ indicates that $x_{j}$ has been excluded, that is,

$$
S_{k}\left(x_{1}, \ldots, \widehat{x}_{j}, \ldots, x_{n}\right)=S_{k}\left(x_{1}, \ldots, x_{j-1}, 0, x_{j+1}, \ldots, x_{n}\right) .
$$

Proof. By differentiation of $S_{k}\left(x_{1}, \ldots, x_{n}\right)$ with respect to $x_{j}$ we get

$$
\frac{\partial}{\partial x_{j}} S_{k}\left(x_{1}, \ldots, x_{n}\right)=S_{k-1}\left(x_{1}, \ldots, \widehat{x}_{j}, \ldots, x_{n}\right),
$$

where $\widehat{x}_{j}$ denotes that $x_{j}$ has been excluded. The proof follows after comparing (2.3) and (2.5).

Proposition 2.4 (Euler's identity). Given any integer $k$, let $S_{k}: \mathbb{R}^{n} \longrightarrow \mathbb{R}$ be the elementary $k$-symmetric polynomial. Then

$$
\sum_{j=1}^{n} x_{j} \frac{\partial}{\partial x_{j}} S_{k+1}\left(x_{1}, \ldots, x_{n}\right)=(k+1) S_{k+1}\left(x_{1}, \ldots, x_{n}\right),
$$

or equivalently,

$$
\sum_{j=1}^{n} x_{j} S_{k}\left(x_{1}, \ldots, \widehat{x}_{j}, \ldots, x_{n}\right)=(k+1) S_{k+1}\left(x_{1}, \ldots, x_{n}\right) .
$$

Proof. See [11].

3. $r$-Newton Operators. Throughout what follows, $V$ stands for an $n$-dimensional real vector space equipped with an inner product. 
DEFINITION 3.1. We define the elementary $r$-symmetric polynomial by

$$
\begin{aligned}
S_{r}: \mathcal{L}(V) & \longrightarrow \mathbb{R} \\
B & \longrightarrow S_{r}(B):=S_{r}\left(\lambda_{1}, \lambda_{2}, \ldots, \lambda_{n}\right),
\end{aligned}
$$

where $B \in \mathcal{L}(V)$ is a self-adjoint linear operator and we let $\lambda_{1}, \ldots, \lambda_{n}$ denote the set of its associated eigenvalues.

Definition 3.2. Let $B \in \mathcal{L}(V)$ be a self-adjoint linear operator and we let $\left\{\lambda_{i}\right\}_{i \in \mathbb{Z}}$ denote all its eigenvalues and let $\left\{v_{i}\right\}_{i \in \mathbb{Z}}$ be its associated orthonormal eigenvectors, i.e., $B v_{i}=\lambda_{i} v_{i}$. We make use of the convention that $\lambda_{i}=0$ if $i \in \mathbb{Z} \backslash\{1,2, \ldots, n\}$ and $v_{i} \begin{cases}\neq 0_{V}, & \text { if } i \in\{1,2, \ldots, n\} ; \\ =0_{V}, & \text { if } i \in \mathbb{Z} \backslash\{1,2, \ldots, n\} .\end{cases}$

We define

$$
B_{i}:= \begin{cases}B, & \forall i \in \mathbb{Z} \backslash\{1,2, \ldots, n\} \\ B_{\left.\right|_{\text {span }\left\{v_{i}\right\}^{\perp}},} & \forall i=1,2, \ldots, n .\end{cases}
$$

Definition 3.3. Let $B \in \mathcal{L}(V)$ be a self-adjoint linear operator. Denote by $B_{i}$ the operator defined in Definition 3.2. We define

$$
S_{r}\left(B_{i}\right):= \begin{cases}0, & \text { if } r \in \mathbb{Z} \backslash\{0,1, \ldots, n\} \text { and } i \in \mathbb{Z} ; \\ 1, & \text { if } r=0 \text { and } i \in \mathbb{Z} ; \\ S_{r}(B), & \text { if } r \in\{1, \ldots, n\} \text { and } i \in \mathbb{Z} \backslash\{1,2, \ldots, n\} ; \\ S_{r}\left(\lambda_{1}, \ldots, \lambda_{i-1}, \widehat{\lambda_{i}}, \lambda_{i+1}, \ldots, \lambda_{n}\right), & \text { if } r \in\{1, \ldots, n\} \text { and } i \in\{1,2, \ldots, n\},\end{cases}
$$

where $\widehat{\lambda_{i}}$ means that the term $\lambda_{i}$ is excluded, that is,

$$
S_{r}\left(\lambda_{1}, \ldots, \lambda_{i-1}, \widehat{\lambda_{i}}, \lambda_{i+1}, \ldots, \lambda_{n}\right)=S_{r}\left(\lambda_{1}, \ldots, \lambda_{i-1}, 0, \lambda_{i+1}, \ldots, \lambda_{n}\right) .
$$

Proposition 3.4. Let $B \in \mathcal{L}(V)$ be a self-adjoint linear operator, $\left\{\lambda_{i}\right\}, 1 \leq i \leq n$, its eigenvalues and let $\left\{v_{i}\right\}, 1 \leq i \leq n$, be its associated orthonormal eigenvectors. Then,

(a) $S_{n}\left(B_{i}\right)=0 ; \forall i \in\{1, \ldots, n\}$;

(b) $S_{r}\left(B_{i}\right)=\frac{\partial}{\partial \lambda_{i}} S_{r+1}(B)$;

(c) $S_{r+1}\left(B_{i}\right)=S_{r+1}(B)-\lambda_{i} S_{r}\left(B_{i}\right)$;

(d) $\frac{\partial}{\partial \lambda_{j}} S_{r}\left(B_{i}\right)=\frac{\partial}{\partial \lambda_{i}} S_{r}\left(B_{j}\right)$.

Proof.

(a) This is immediate from the fact that $S_{n}\left(B_{i}\right)$ is a product of $n$ terms where one of them is zero.

(b) Immediate from Definition 3.3 and equations (2.3) and (2.4).

(c) In the expression (2.2), we apply Definition 3.3 and the above item (b).

(d) This is a consequence of the item (b).

DEFINITION 3.5. Given any integer $r$, an operator

$$
P_{r}:\{B \in \mathcal{L}(V) ; B \text { is self-adjoint }\} \longrightarrow\{B \in \mathcal{L}(V) ; B \text { is self-adjoint }\}
$$


given by

$$
P_{r}(B):= \begin{cases}I, & r=0 ; \\ \sum_{j=0}^{r}(-1)^{j} S_{r-j}(B) B^{j}, & \forall r \in\{1,2, \ldots, n-1\} ; \\ \mathcal{O}, & r \in \mathbb{Z} \backslash\{0,1, \ldots, n\} .\end{cases}
$$

is called an $r$-Newton operator associated to $B$, where $I$ and $\mathcal{O}$ are the identity and the null operators, respectively.

PRoposition 3.6.

$$
P_{r+1}(B)=S_{r+1}(B) I-B P_{r}(B)
$$

or equivalently,

$$
P_{r+1}(B)=S_{r+1}(B) I-P_{r}(B) B
$$

for each $r=0,1,2, \ldots, n-1$.

Proof. The proof of (3.1) is by induction on $r$ and (3.2) is justified by the fact that $P_{r}(B)$ is a polynomial and $P_{r}(B) B=B P_{r}(B)$.

The next proposition is a summary of important relations about $P_{r}(B)$ and $S_{r}(B)$.

Proposition 3.7. Let $B \in \mathcal{L}(V)$ be a self-adjoint operator. Then

(a) $P_{n}(B)=\mathcal{O}$, where $\mathcal{O}$ is the null operator in $V$;

(b) trace $\left(B P_{r}(B)\right)=n S_{r+1}(B)-\operatorname{trace}\left(P_{r+1}(B)\right)$;

(c) trace $\left(B^{2} P_{r}(B)\right)=\operatorname{trace}\left(S_{r+1}(B) B\right)-\operatorname{trace}\left(B P_{r+1}(B)\right)$;

(d)

(d.1) trace $\left(P_{r}(B)\right)=(n-r) S_{r}(B)$;

(d.2) trace $\left(P_{r}(B)\right)=\sum_{j=0}^{r}(-1)^{j} S_{r-j}(B)$ trace $\left(B^{j}\right)$;

(e) trace $\left(B P_{r}(B)\right)=(r+1) S_{r+1}(B) \quad$ (Newton's formula);

(f) trace $\left(B^{2} P_{r}(B)\right)=S_{1}(B) S_{r+1}(B)-(r+2) S_{r+2}(B)$;

(g) $P_{r}(B)$ and $B$ have the same eigenvectors;

(h) The eigenvalues of $P_{r}(B)$ are $\frac{\partial}{\partial \lambda_{j}} S_{r}(B)$, where $\lambda_{j}$ is an eigenvalue of $B$;

(i) $P_{r}(B) v_{i}=S_{r}\left(B_{i}\right) v_{i}$, where $v_{i}$ is an eigenvector of $B$;

(j) trace $\left(P_{r}(B)\right)=\sum_{i=1}^{n} S_{r}\left(B_{i}\right)$;

(k) $\operatorname{trace}\left(B P_{r}(B)\right)=\sum_{i=1}^{n} \lambda_{i} S_{r}\left(B_{i}\right)$;

(1) $\operatorname{trace}\left(B^{2} P_{r}(B)\right)=\sum_{i=1}^{n} \lambda_{i}^{2} S_{r}\left(B_{i}\right)$.

Proof. See [3]. 
Definition 3.8. Let $B \in \mathcal{L}(V)$ be a self-adjoint linear operator. Denote by $B_{i}$ the operator given in Definition 3.2. We define

$$
S_{r}\left(B_{i}, B_{j}\right):= \begin{cases}0 & \text { if } r \in \mathbb{Z} \backslash\{0,1, \ldots, n\}, \forall i, j \in \mathbb{Z} ; \\ 1 & \text { if } r=0, \forall i, j \in \mathbb{Z} ; \\ S_{r}(B) & \text { if } r \in\{1, \ldots, n\}, \forall i, j \in \mathbb{Z} \backslash\{1,2, \ldots, n\} ; \\ S_{r}\left(B_{i}\right) & \text { if } r, i \in\{1, \ldots, n\}, j \in \mathbb{Z} \backslash\{1,2, \ldots, n\} ; \\ S_{r}\left(B_{j}\right) & \text { if } r, j \in\{1, \ldots, n\}, i \in \mathbb{Z} \backslash\{1,2, \ldots, n\} ; \\ S_{r}\left(B_{i}\right) \quad & \text { if } r \in\{1, \ldots, n\}, j=i \in\{1,2, \ldots, n\} ; \\ S_{r}\left(\lambda_{1}, \ldots, \lambda_{i}, \ldots, \widehat{\lambda_{j}}, \ldots, \lambda_{n}\right) & \text { if } r \in\{1, \ldots, n\}, j \neq i \in\{1,2, \ldots, n\} .\end{cases}
$$

where $\widehat{\lambda_{i}}$ means that the term $\lambda_{i}$ is excluded and we are denoting

$$
S_{r}\left(B_{i}, B_{j}\right):=S_{r}\left(B_{\left.\right|_{\text {span }\left\{v_{i}, v_{j}\right\}^{\perp}}}\right) .
$$

Note that $S_{r}\left(B_{i}, B_{j}\right)$ is an extension of Definition 3.3. We next show a few relations involving $S_{r}\left(B_{i}, B_{j}\right)$.

Proposition 3.9. Let $B \in \mathcal{L}(V)$ be a self-adjoint operator, $\lambda_{1}, \ldots, \lambda_{n}$ the eigenvalues of $B$ and let $v_{1}, \ldots, v_{n}$ be its associated orthonormal eigenvectors. Then

(a) $S_{r}\left(B_{i}, B_{j}\right)=S_{r}\left(B_{j}, B_{i}\right)$;

(b) $S_{r}\left(B_{i}, B_{j}\right)=\frac{\partial^{2}}{\partial \lambda_{i} \partial \lambda_{j}} S_{r+2}(B), \quad \forall i, j \in\{1, \ldots, n\}$;

(c) $S_{n-1}\left(B_{i}, B_{j}\right)=0=S_{n}\left(B_{i}, B_{j}\right), \quad \forall i, j \in\{1, \ldots, n\}$;

(d)

$$
S_{r+1}\left(B_{i}, B_{j}\right)=S_{r+1}\left(B_{i}\right)-\lambda_{j} S_{r}\left(B_{i}, B_{j}\right)
$$

and

$$
S_{r+1}\left(B_{i}, B_{j}\right)=S_{r+1}\left(B_{j}\right)-\lambda_{i} S_{r}\left(B_{i}, B_{j}\right) ;
$$

(e) $S_{r+1}\left(B_{i}\right)-S_{r+1}\left(B_{j}\right)=\left(\lambda_{j}-\lambda_{i}\right) S_{r}\left(B_{i}, B_{j}\right)$;

(f) $\sum_{\substack{i=1 \\ i \neq k}}^{n} \lambda_{i} S_{r}\left(B_{i}, B_{k}\right)=(r+1) S_{r+1}\left(B_{k}\right) \quad$ (Euler's identity);

(g) With the definition of $S_{r}\left(B_{i}, B_{j}, B_{k}\right)$, we have (analogous to part (d)):

$$
\begin{aligned}
& S_{r+1}\left(B_{i}, B_{j}, B_{k}\right)=S_{r+1}\left(B_{i}, B_{j}\right)-\lambda_{k} S_{r}\left(B_{i}, B_{j}, B_{k}\right), \\
& S_{r+1}\left(B_{i}, B_{j}, B_{k}\right)=S_{r+1}\left(B_{i}, B_{k}\right)-\lambda_{j} S_{r}\left(B_{i}, B_{j}, B_{k}\right), \\
& S_{r+1}\left(B_{i}, B_{j}, B_{k}\right)=S_{r+1}\left(B_{j}, B_{k}\right)-\lambda_{i} S_{r}\left(B_{i}, B_{j}, B_{k}\right) ;
\end{aligned}
$$

(h) $S_{r+1}\left(B_{i}, B_{k}\right)-S_{r+1}\left(B_{k}, B_{j}\right)=\left(\lambda_{j}-\lambda_{i}\right) S_{r}\left(B_{i}, B_{j}, B_{k}\right)$; 
(i) For any $j \in\{1, \ldots, n\}, r \in\{0, \ldots, n\}$ we have 3

$$
[(n-1)-r] S_{r}\left(B_{j}\right)=\sum_{\substack{i=1 \\ i \neq j}}^{n} S_{r}\left(B_{i}, B_{j}\right) .
$$

Proof.

(a) Direct from the equality

$$
S_{r}\left(B_{i}, B_{j}\right)=S_{r}\left(\left(B_{\left.\right|_{\text {span }\{v i\}^{\perp}}}\right)_{\left.\right|_{\operatorname{span}\left\{v_{j}\right\}^{\perp}}}\right)=S_{r}\left(\left(\left.B\right|_{\operatorname{span}\left\{v_{j}\right\rangle^{\perp}}\right)_{\left.\right|_{\text {span }\left\{v_{i}\right\rfloor^{\perp}}}\right) .
$$

(b) We have

$$
\begin{aligned}
\frac{\partial^{2}}{\partial \lambda_{i} \partial \lambda_{j}} S_{r+2}(B) & =\frac{\partial}{\partial \lambda_{i}}\left(\frac{\partial}{\partial \lambda_{j}} S_{r+2}(B)\right), \\
& =\frac{\partial}{\partial \lambda_{i}}\left(S_{r+1}\left(B_{\left.\right|_{\operatorname{span}\left\{e_{j}\right\}^{\perp}}}\right)\right)=S_{r}\left(\left(B_{\left.\right|_{\text {span }\{e i\}^{\perp}}}\right)_{\left.\right|_{\text {span }\left\{e_{j}\right\}^{\perp}}}\right) .
\end{aligned}
$$

The last equality comes from Proposition 3.4(b) and Definition 3.2.

(c) It is immediate from part (b) and Proposition 3.4(b).

(d) Use that the statement of Proposition 3.4(c) remains valid if we replace $B$ by $B_{\left.\right|_{\text {span }\left\{v_{j}\right\}^{\perp}} \text {. }}$

(e) Immediate from part (d).

(f) First use notation of Definition 3.3 in relation (2.7) and finally replace $B$ by $B_{\left.\right|_{\text {span }\left\{v_{j}\right\}^{\perp}} \text {. }}$

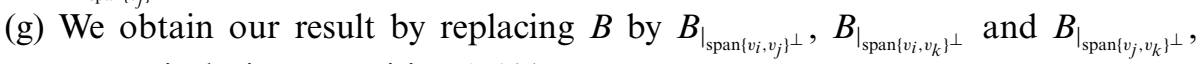
successively, in Proposition 3.4(c).

(h) Immediate from part (g).

(i) Only replace $B$ by $B_{\left.\right|_{\text {span }\left\{v_{j}\right\}^{\perp}}}$ in Proposition 3.7(e) and (k).

4. First Applications. Here, we give some applications of the result obtained in Section 3, including another proof of a result of Fialkow in [8] about Einstein manifolds. A Riemannian manifold $\mathbf{M}^{n}$ is an Einstein manifold if its Ricci tensor satisfies: for any $X, Y$ tangent to $M^{n}, \operatorname{Ric}(X, Y)=\lambda\langle X, Y\rangle$, where $\lambda$ is a real function.

THEOREM 4.1 ([8] part of Theorem 7.1). Let $\mathbf{M}^{n}$ be a connected Riemannian manifold and $x: \mathbf{M}^{n} \longrightarrow \overline{\mathbf{M}}^{n+1}(c), n \geq 3$, an isometric immersion. If $\mathbf{M}^{n}$ is an Einstein manifold, then the maximum number of distinct principal curvatures of $x$ is two.

Proof. From Gauss equation

$$
\operatorname{Ric}(X, Y)=c(n-1)\langle X, Y\rangle+\left\langle A P_{1}(A) X, Y\right\rangle,
$$

${ }^{3}$ This is a version of Proposition 3.7 (d.1). 
where $A$ is the shape operator. Let $\left\{e_{1}, \ldots, e_{n}\right\}$ be an orthonormal basis which diagonalizes $A$ at a point (that is, $A e_{i}=\lambda_{i} e_{i}$ ). Since $\mathbf{M}^{n}$ is Einstein we get

$$
\lambda=c(n-1)+\lambda_{i} S_{1}\left(A_{i}\right), \quad \text { for } i=1,2, \ldots, n .
$$

If $\lambda_{1}=\cdots=\lambda_{n}=\rho$, clearly $\lambda_{i} S_{1}\left(A_{i}\right)=(n-1) \rho^{2}$. From (4.1) we get $\rho^{2}=\frac{\lambda}{n-1}-c$. Since $\rho \in \mathbb{R}$ it follows that

(a) $\lambda>c(n-1)$ or

(b) $\lambda=c(n-1)$.

If $x$ has at least two distinct principal curvatures $\lambda_{i} \neq \lambda_{j}$, from (4.1) we have that $\lambda_{i} S_{1}\left(A_{i}\right)=\lambda_{j} S_{1}\left(A_{j}\right)$; from Proposition 3.4(c) we get $S_{2}\left(A_{j}\right)-S_{2}\left(A_{i}\right)=0$. From Proposition 3.9(d) we get $\left(\lambda_{i}-\lambda_{j}\right) S_{1}\left(A_{i}, A_{j}\right)=S_{2}\left(A_{j}\right)-S_{2}\left(A_{i}\right)=0$. Then $S_{1}\left(A_{i}, A_{j}\right)=0$. By Proposition 3.9(d) we get $S_{1}\left(A_{i}\right)=S_{1}\left(A_{i}, A_{j}\right)+\lambda_{j}$ and hence $\lambda_{i} S_{1}\left(A_{i}\right)=$ $\lambda_{i} S_{1}\left(A_{i}, A_{j}\right)+\lambda_{i} \lambda_{j}$. But we see that $S_{1}\left(A_{i}, A_{j}\right)=0$ and so $\lambda_{i} S_{1}\left(A_{i}\right)=\lambda_{i} \lambda_{j}$. In other words, from (4.1) we get $\lambda_{i} \lambda_{j}=\lambda-c(n-1)$.

Now, for any principal curvature $\lambda_{k}$ with $k \neq i, j$, by (4.1) we have

$$
\lambda_{k} S_{1}\left(A_{k}\right)=\lambda-c(n-1) \text {. }
$$

From Proposition 3.4(c) we get

$$
\lambda_{k}\left(S_{1}(A)-\lambda_{k}\right)=\lambda-c(n-1) .
$$

We have seen that $\lambda_{i} \lambda_{j}=\lambda-c(n-1)$. Thus

$$
\lambda_{k}^{2}-S_{1}(A) \lambda_{k}+\lambda_{i} \lambda_{j}=0 .
$$

Since $S_{1}(A)=S_{1}\left(A_{i}, A_{j}\right)+\lambda_{i} \lambda_{j}$, and $S_{1}\left(A_{i}, A_{j}\right)=0$ we get

$$
\lambda_{k}^{2}-\left(\lambda_{i}+\lambda_{j}\right) \lambda_{k}+\lambda_{i} \lambda_{j}=0 .
$$

Therefore, the above equality shows that each $\lambda_{k}$ must be $\lambda_{i}$ or $\lambda_{j}$.

THEOREM 4.2. Let $B \in \mathcal{L}(V)$ be a self-adjoint linear operator. For any $r, k \in \mathbb{Z}$ we have

$$
\operatorname{trace}\left[P_{r-1}(B) B P_{k}(B)\right]=\sum_{j=0}^{k}(r+k-2 j) S_{r+k-j}(B) S_{j}(B) .
$$

Proof. Multiply relation (3.2) on both sides by $P_{k}(B)$ to get

$$
P_{r}(B) P_{k}(B)=S_{r}(B) P_{k}(B)-P_{r-1}(B) B P_{k}(B) .
$$

Next, taking trace and by Proposition 3.7(d.1) we get

$$
\operatorname{trace}\left(P_{r}(B) P_{k}(B)\right)=(n-k) S_{r}(B) S_{k}(B)-\operatorname{trace}\left(P_{r-1}(B) B P_{k}(B)\right) .
$$

It follows, by interchanging the roles of $r$ and $k$, that

$$
\operatorname{trace}\left(P_{k}(B) P_{r}(B)\right)=(n-r) S_{k}(B) S_{r}(B)-\operatorname{trace}\left(P_{k-1}(B) B P_{r}(B)\right) .
$$


Hence,

$$
(r-k) S_{r}(B) S_{k}(B)=\operatorname{trace}\left(P_{r-1}(B) B P_{k}(B)\right)-\operatorname{trace}\left(P_{k-1}(B) B P_{r}(B)\right) .
$$

For fixed $r$, the proof is by induction on $k$.

Taking $k=1$ in (4.2), by Proposition 3.7(d.1) we get

$$
\operatorname{trace}\left(P_{r-1}(B) B P_{1}(B)\right)=(r+1) S_{r+1}(B)+(r-1) S_{1}(B) S_{r}(B) .
$$

By the induction hypothesis, we then have

$$
\operatorname{trace}\left[P_{r-1}(B) B P_{k}(B)\right]=\sum_{j=0}^{k}(r+k-2 j) S_{r+k-j}(B) S_{j}(B) .
$$

Replacing $k$ for $k+1$ in (4.2) we get

$$
\operatorname{trace}\left[P_{r-1}(B) B P_{k+1}(B)\right]=(r-k-1) S_{r}(B) S_{k+1}(B)+\operatorname{trace}\left[P_{k}(B) B P_{r}(B)\right] \text {. }
$$

Again by the induction hypothesis, we then have

$$
\begin{aligned}
& \operatorname{trace}\left[P_{r-1}(B) B P_{k+1}(B)\right]= \\
& \quad(r-k-1) S_{r}(B) S_{k+1}(B)+\sum_{j=0}^{k}(r+1+k-2 j) S_{r+1+k-j}(B) S_{j}(B) .
\end{aligned}
$$

Therefore,

$$
\operatorname{trace}\left[P_{r-1}(B) B P_{k+1}(B)\right]=\sum_{j=0}^{k+1}(r+1+k-2 j) S_{r+1+k-j}(B) S_{j}(B) .
$$

From now onwards $\|\cdot\|$ means the Hilbert-Schmidt norm, that is, if $B$ is any linear operator

$$
\|B\|:=\sqrt{\operatorname{trace}\left(B^{*} \circ B\right)}
$$

where $B^{*}$ means the adjoint to the operator $B$.

COROllary 4.3. Let $B \in \mathcal{L}(V)$ be a self-adjoint linear operator. For any $r \in \mathbb{Z}$ we have

$$
\left\|P_{r}(B)\right\|^{2}=(n-r) S_{r}(B)^{2}-2 \sum_{j=0}^{r-1}(r-j) S_{j}(B) S_{2 r-j}(B) .
$$

Proof. Multiply relation (3.2) on the right by $P_{r}(B)$ to get

$$
P_{r}(B) P_{r}(B)=S_{r}(B) P_{r}(B)-P_{r-1}(B) B P_{r}(B) .
$$

Now the proof follows as a consequence of taking the trace and also by Proposition 3.7(d.1) and by Theorem 4.2. 

have

COROLlary 4.4. Let $B \in \mathcal{L}(V)$ be a self-adjoint linear operator. For any $r \in \mathbb{Z}$ we

$$
\left\|B P_{r-1}(B)\right\|^{2}=r S_{r}(B)^{2}-2 \sum_{j=0}^{r-1}(r-j) S_{j}(B) S_{2 r-j}(B) .
$$

Proof. By (3.1),

$$
P_{r}(B)^{2}=B P_{r-1}(B) B P_{r-1}(B)-2 S_{r}(B) B P_{r-1}(B)+S_{r}(B)^{2} I .
$$

Now take the trace of this expression. Then, by Proposition 3.7(e) and since $B P_{r-1}(B)=$ $P_{r-1}(B) B$, we have

$$
\left\|P_{r}(B)\right\|^{2}=\left\|B P_{r-1}(B)\right\|^{2}+(n-2 r) S_{r}(B)^{2} .
$$

Corollary 4.3 finishes the proof.

5. $k$-umbilicity in Riemannian Manifolds. Let $\mathbf{M}^{n}$ and $\overline{\mathbf{M}}^{n+1}$ be Riemannian manifolds of dimension $n$ and $n+1$, respectively. Let $x: \mathbf{M}^{n} \longrightarrow \overline{\mathbf{M}}^{n+1}$ be an isometric immersion and denote its shape operator at a point $q$ in $\mathbf{M}^{n}$ by $A: T_{q} \mathbf{M} \rightarrow T_{q} \mathbf{M}$ (by abuse of language $A$ is also called the second fundamental form).

Definition 5.1. An isometric immersion $x: \mathbf{M}^{n} \longrightarrow \overline{\mathbf{M}}^{n+1}$ is said to be $k$-umbilical at $q \in \mathbf{M}^{n}, k=1, \ldots, n-1$, if

$$
A P_{k-1}(A)=\lambda I,
$$

where $\lambda=\lambda(k)$ is a real function and $I$ is the identity map of $T_{q} \mathbf{M}$.

Even though we do not know any term in (5.1), by Proposition 3.7(e) we can show that $\lambda(k)=\frac{k}{n} S_{k}(A)$. Hence, another way to define $k$-umbilicity is

$$
A P_{k-1}(A)=\frac{k}{n} S_{k}(A) I .
$$

By Proposition 3.6, we can get an equivalent definition of $k$-umbilicity:

$$
P_{k}(A)=\left(1-\frac{k}{n}\right) S_{k}(A) I .
$$

Definition 5.2. We say that an isometric immersion $x: \mathbf{M}^{n} \longrightarrow \overline{\mathbf{M}}^{n+1}$ is $k$-umbilical when it is $k$-umbilical at every point of $\mathbf{M}$.

Definition 5.3. An isometric immersion $x: \mathbf{M}^{n} \longrightarrow \overline{\mathbf{M}}^{n+1}$ is said to be $k$-totally geodesic if

$$
A P_{k-1}(A)=0
$$

Proposition 5.4. Let $x: \mathbf{M}^{n} \longrightarrow \overline{\mathbf{M}}^{n+1}$ be an isometric immersion. $x$ is $k$-totally geodesic if and only if $x$ is $k$-umbilical with $S_{k}(A)=0$. 
Proof. Let $x$ be $k$-umbilical such that $S_{k}(A)=0$. Then by (5.2) $x$ is $k$-totally geodesic.

Conversely, let $x$ be $k$-totally geodesic. Then $A P_{k-1}(A)=0=0 I$. Thus every $k$-totally geodesic is $k$-umbilical. By Proposition 3.7(e) we have that $S_{k}(A)=0$.

It is known that the second fundamental form $A$ of a totally umbilical isometric immersion $x: \mathbf{M}^{n} \longrightarrow \overline{\mathbf{M}}^{n+1}$ satisfies the Codazzi equation if and only if $S_{1}$ is constant. We generalize this fact for $k$-umbilical isometric immersions with $A P_{k-1}(A)$ in place of $A$. As a $(1,1)$ symmetric tensor, $A P_{k-1}(A)$ is said to be Codazzi if $\left(\nabla_{X} A P_{k-1}(A)\right) Y=$ $\left(\nabla_{Y} A P_{k-1}(A)\right) X$, where

$$
\left(\nabla_{X} A P_{k-1}(A)\right)(Y)=\nabla_{Y}\left(A P_{k-1}(A)(X)\right)-A P_{k-1}(A)\left(\nabla_{X} Y\right),
$$

for any $X, Y$ tangents to $\mathbf{M}$.

THeOrem 5.5. Let $\mathbf{M}^{n}$ be a connected Riemannian manifold and $x: \mathbf{M}^{n} \longrightarrow \overline{\mathbf{M}}^{n+1}$ a k-umbilical isometric immersion. Then

$$
A P_{k-1}(A) \text { is Codazzi if and only if } S_{k}(A) \text { is constant. }
$$

Proof. For any $X, Y$ tangents to $\mathbf{M}^{n}$

$$
\left(\nabla_{X} A P_{k-1}(A)\right)(Y)=\nabla_{Y}\left(A P_{k-1}(A)(X)\right)-A P_{k-1}(A)\left(\nabla_{X} Y\right) .
$$

Since the immersion is $k$-umbilical

$$
\left(\nabla_{X} A P_{k-1}(A)\right)(Y)-\left(\nabla_{Y} A P_{k-1}(A)\right)(X)=X\left(\frac{k}{n} S_{k}(A)\right) Y-Y\left(\frac{k}{n} S_{k}(A)\right) X .
$$

If $A P_{k-1}(A)$ is Codazzi, then the left-hand side of (5.4) is zero. Hence,

$$
X\left(\frac{k}{n} S_{k}(A)\right) Y-Y\left(\frac{k}{n} S_{k}(A)\right) X=0 .
$$

Now if $X$ and $Y$ are chosen to be linearly independent, we get

$$
X\left(\frac{k}{n} S_{k}(A)\right)=0=Y\left(\frac{k}{n} S_{k}(A)\right)
$$

thus, $\frac{k}{n} S_{k}(A)$ is constant at every point of $\mathbf{M}^{n}$ and by the connectedness of $\mathbf{M}^{n}$ it follows that $\frac{k}{n} S_{k}(A)$ is constant in $\mathbf{M}^{n}$. Thus,

Conversely, if $\frac{k}{n} S_{k}(A)$ is constant in $\mathbf{M}^{n}$, then the right-hand side of (5.4) is zero.

$$
\left(\nabla_{X} A P_{k-1}(A)\right)(Y)-\left(\nabla_{Y} A P_{k-1}(A)\right)(X)=0 .
$$

Hence, $A P_{k-1}(A)$ is Codazzi.

Now we are going to introduce an operator, defined on tangent spaces, which measures how much an isometric immersion fails to be $k$-umbilical. 
Definition 5.6. Let $x: \mathbf{M}^{n} \longrightarrow \overline{\mathbf{M}}^{n+1}$ be an isometric immersion and $A$ its shape operator. For each $p \in \mathbf{M}$, the $k$-umbilicity operator

$$
\phi_{k}: T_{p} \mathbf{M} \longrightarrow T_{p} \mathbf{M}
$$

is defined by

$$
\phi_{k}(X):=\frac{k}{n} S_{k}(A) X-A P_{k-1}(A) X, \quad \forall X \in T_{p} \mathbf{M},
$$

where $P_{i}$ is the $i$ th Newton operator and $S_{j}(A)$ is the $j$ th symmetric function associated to $A$.

REMARK 5.7. We must note that when $k=1$ the operator $\phi_{1}(X)=\phi(X)=H X-$ $A X$ was used in [1], where $\phi_{1} \equiv 0$ if and only if the immersion is totally umbilical. This fact extends to $k$-umbilical immersions: by (5.2) $\phi_{k} \equiv 0$ if and only if the immersion is $k$-umbilical; in another words, the operator $\phi_{k}$ gives a measure of how much an isometric immersion fails to be $k$-umbilical.

Proposition 5.8. Let $x: \mathbf{M}^{n} \longrightarrow \overline{\mathbf{M}}^{n+1}$ be an isometric immersion and let $A$ be its shape operator. Then the map $\phi_{k}$ satisfies the following:

(a) $\phi_{k}$ is self-adjoint;

(b) $\phi_{k}$ is simultaneously diagonalizable with $A$ and if $\left\{e_{1}, \ldots, e_{n}\right\}$ is an orthonormal basis which diagonalizes $A$ we have $\phi_{k}\left(e_{i}\right)=\mu_{i} e_{i}$, where

$$
\mu_{i}=S_{k}\left(A_{i}\right)-\left(1-\frac{k}{n}\right) S_{k}
$$

Proof.

(a) Since $A$ is self-adjoint, it follows that $\phi_{k}$ is self-adjoint, too.

(b) The proof follows by using that $A e_{i}=\lambda_{i} e_{i}, i=1,2, \ldots, n$ and Proposition 3.7(i).

Proposition 5.9. Let $x: \mathbf{M}^{n} \longrightarrow \overline{\mathbf{M}}^{n+1}$ be an isometric immersion and let $A$ be its shape operator. Let $\phi_{k}$ be given by the Definition 5.6 and $\|$.$\| denotes the Hilbert-Schmidt$ norm. Then

1. $\left\|\phi_{k}\right\|^{2}=\sum_{i=1}^{n} \mu_{i}^{2}$, where $\mu_{i}$ was defined in Proposition 5.8(b);

2. $\left\|\phi_{k}\right\|^{2}=\frac{k(n-k)}{n} S_{k}(A)^{2}-2 \sum_{j=0}^{k-1}(k-j) S_{j}(A) S_{2 k-j}(A)$;

3. $\left\|\phi_{k}\right\|^{2}=\frac{1}{n} \sum_{1 \leq i<j \leq n}\left(\lambda_{i} S_{k-1}\left(A_{i}\right)-\lambda_{j} S_{k-1}\left(A_{j}\right)\right)^{2}$.

Proof. Let $\left\{e_{1}, \ldots, e_{n}\right\}$ be an orthonormal basis such that $A e_{i}=\lambda_{i} e_{i}$ for $i=$ $1, \ldots, n$. Here,

$$
\left\|\phi_{k}\right\|^{2}=\operatorname{trace}\left(\phi_{k}^{*} \circ \phi_{k}\right)
$$

where $\phi_{k}^{*}$ is the adjoint of $\phi_{k}$. 
1. The proof follows from Proposition 5.8(a) and (b).

2. By part (1), Proposition 5.8(b) and Proposition 3.7(j) and (d.1) we get

$$
\left\|\phi_{k}\right\|^{2}=\sum_{i=1}^{n} S_{k}\left(A_{i}\right)^{2}-\frac{(n-k)^{2}}{n} S_{k}(A)^{2}
$$

in another words,

$$
\left\|\phi_{k}\right\|^{2}=\operatorname{trace}\left(P_{k}(A)^{2}-\frac{(n-k)^{2}}{n^{2}} S_{k}(A)^{2} I\right) .
$$

From Propositions 3.6 and 3.7 we get

$$
\left\|\phi_{k}\right\|^{2}=\left((n-k) S_{k}(A)^{2}-\operatorname{trace}\left(P_{k-1}(A) A P_{k}(A)\right)\right)-\frac{(n-k)^{2}}{n} S_{k}(A)^{2} .
$$

Finally use Theorem 4.2.

3. We can see that $2 S_{2}\left(A P_{k-1}(A)\right)=S_{1}\left(A P_{k-1}(A)\right)^{2}-\left\|A P_{k-1}(A)\right\|^{2}$. Our result then follows from Proposition 3.7(e) and Corollary 4.4.

6. Characterizations of $k$-umbilical immersions. Here, $\overline{\mathbf{M}}^{n+1}$ will be a Riemannian manifold.

Proposition 6.1. Let $x: \mathbf{M}^{n} \longrightarrow \overline{\mathbf{M}}^{n+1}$ be an isometric immersion, $A$ its shape operator and $\left\{e_{1}, \ldots, e_{n}\right\}$ an orthonormal basis which diagonalizes $A$ at a point $q \in M$. The immersion is $k$-umbilical at $q$ if and only if in Newton's Formula, $\sum_{j=1}^{n} \lambda_{j} S_{k-1}\left(A_{j}\right)=$ $k S_{k}(A)$, each term in the sum is equal to $\frac{k}{n} S_{k}(A)$ at $q$.

Proof. The proof follows by using (5.2) and Proposition 3.7(i).

REMARK 6.2. We see from above that an isometric immersion $x: \mathbf{M}^{n} \longrightarrow \overline{\mathbf{M}}^{n+1}$ is $k$-umbilical if and only if

$$
\lambda_{j} S_{k-1}\left(A_{j}\right)=\frac{k}{n} S_{k}(A), \forall j
$$

Corollary 6.3. Let $x: \mathbf{M}^{n} \longrightarrow \overline{\mathbf{M}}^{n+1}$ be an isometric immersion, $A$ its shape operator and $\left\{e_{1}, \ldots, e_{n}\right\}$ an orthonormal basis which diagonalizes $A$ at $q \in M$. The immersion is $k$-umbilical if and only if

$$
\sum_{j=0}^{k-1}(-1)^{j} S_{j}(A) \lambda_{i}^{k-j}+(-1)^{k} \frac{k}{n} S_{k}(A)=0 \quad \text { at } q \in \mathbf{M}
$$

Proof. From definition of $A_{i}$ and (2.4) we get

$$
S_{k}\left(A_{i}\right)=\sum_{j=0}^{k}(-1)^{j} S_{k-j}(A)\left(\lambda_{i}\right)^{j} .
$$

The proof now follows from identity (6.2) and Remark 6.2. 
Remark 6.4. Let $x: \mathbf{M}^{n} \longrightarrow \overline{\mathbf{M}}^{n+1}$ be an isometric immersion. Then $x$ is one-umbilical if and only if $\lambda_{i}=\frac{1}{n} S_{1}$; $x$ is two-umbilical if and only if $\lambda_{i}^{2}-S_{1} \lambda_{i}+\frac{2}{n} S_{2}=0$; $x$ is three-umbilical if and only if $\lambda_{i}^{3}-S_{1} \lambda_{i}^{2}+S_{2} \lambda_{i}-\frac{3}{n} S_{3}=0$.

7. Consequences of the $k$-umbilicity. It is clear that every one-umbilical immersion is a $k$-umbilical immersion, but the converse is not true.

TheOrem 7.1. Let $x: \mathbf{M}^{n} \longrightarrow \overline{\mathbf{M}}^{n+1}$ be an isometric immersion and let $A$ be its shape operator. If $x$ is $k$-umbilical at $q \in \mathbf{M}$, then

$$
H_{k+1}=H_{1} H_{k} \text { at } q \text {. }
$$

The converse is true if all its principal curvatures are different from zero. Moreover, we have the following identity

$$
S_{1}(A) S_{k+1}(A)-(k+2) S_{k+2}(A)=\left(\frac{n-k}{n}\right) S_{k}(A)\|A\|^{2} .
$$

Proof. Let $\left\{e_{1}, \ldots, e_{n}\right\}$ be an orthonormal basis which diagonalizes $A$ at $q$ and $\lambda_{i}$ the eigenvalue corresponding to $e_{i}$. From the $k$-umbilicity of $x$ and Remark 6.2 we have $\lambda_{j}^{2} S_{k-1}\left(A_{j}\right)=\frac{k}{n} S_{k}(A) \lambda_{j}$. By summing on $j$ we have $\sum_{j=1}^{n} \lambda_{j}^{2} S_{k-1}(A)=$ $\frac{k}{n} S_{k}(A)\left(\sum_{j=1}^{n} \lambda_{j}\right)$. Using Proposition 3.7(f) and (1) we get

$$
S_{1}(A) S_{k}(A)-(k+1) S_{k+1}(A)=\frac{k}{n} S_{1}(A) S_{k}(A)
$$

or equivalently

$$
\left(\begin{array}{c}
n \\
k+1
\end{array}\right) \frac{S_{k+1}(A)}{\left(\begin{array}{c}
n \\
k+1
\end{array}\right)}=\frac{n-k}{k+1}\left(\frac{S_{1}(A)}{n}\right) \frac{S_{k}(A)}{\left(\begin{array}{l}
n \\
k
\end{array}\right)}\left(\begin{array}{l}
n \\
k
\end{array}\right) .
$$

Hence,

$$
H_{k+1}=H_{1} H_{k} .
$$

For the converse, suppose that there is a point $q \in M$ such that $H_{k+1}=H_{1} H_{k}$ and $\lambda_{i} \neq 0$ for each $i$ at $q$. Thus,

$$
(k+1) S_{k+1}(A)=\left(\frac{n-k}{n}\right) S_{1}(A) S_{k}(A) ;
$$

reordering the last equality and making use of Proposition 3.7(f) we can rewrite it as

$$
\operatorname{trace}\left(A^{2} P_{k-1}(A)\right)=\frac{k}{n} S_{1}(A) S_{k}(A)
$$


by Proposition 3.7(1), we get

$$
\sum_{j=1}^{n}\left(\lambda_{j}^{2} S_{k-1}\left(A_{j}\right)-\lambda_{j} \frac{k}{n} S_{k}(A)\right)=0 .
$$

Now, let $\left\{v_{1}, \ldots, v_{n}\right\}$ be a basis of $T_{q} M$ and consider the following linear combination:

$$
\sum_{j=1}^{n}\left(\lambda_{j}^{2} S_{k-1}\left(A_{j}\right)-\lambda_{j} \frac{k}{n} S_{k}(A)\right) v_{j}=0 .
$$

Thus, $\lambda_{j}\left(\lambda_{j} S_{k-1}\left(A_{j}\right)-\frac{k}{n} S_{k}(A)\right)=0$, for each $j$. The proof now follows from Remark 6.2.

Proposition 7.2. Every k-umbilical isometric immersion $x: \mathbf{M}^{n} \longrightarrow \overline{\mathbf{M}}^{n+1}$ with a zero principal curvature at a point $p$ is $k$-totally geodesic at $p$ and has at least $n-k+1$ principal curvatures equal to zero at $p$.

Proof. First we will prove that at $p$

$$
S_{j}(A)=0, \quad \forall j \geq k \text { and } S_{j}\left(A_{i}\right)=0, \quad \forall j \geq k, \forall i .
$$

By hypothesis we can suppose $\lambda_{\alpha}=0$, for some $\alpha$. Substitute $\lambda_{i}$ for $\lambda_{\alpha}=0$ into (6.1) to obtain $S_{k}(A)=0$. Since $H_{k+1}=H_{1} H_{k}$, it follows that $S_{k+1}(A)=0$. From (7.1) we have $S_{k+2}(A)=0$. Now, by Propositions 6.1 and 3.4(c) we get $S_{k}\left(A_{i}\right)=0, \forall i$; again by Proposition 3.4(c), we have $S_{k+1}\left(A_{i}\right)=0, \forall i$ and thus $S_{k+2}\left(A_{i}\right)=0, \forall i$. The proof of (7.2) follows from a recursive process using the same arguments.

Now, we are going to show that there exist at least $n-k+1$ principal curvatures equal to zero. We had seen that $S_{n}(A)=0$; then at least one principal curvature is null; denote it by $\lambda_{j_{1}}=\lambda_{\alpha}=0$. We also had seen that $S_{n-1}\left(A_{j}\right)=0$, for any $j$. Thus

$$
S_{n-1}\left(A_{j_{1}}\right)=\prod_{\substack{i=1 \\ i \neq j_{1}}}^{n} \lambda_{i}=0,
$$

and hence there exists another null principal curvature and we denote it by $\lambda_{j_{2}}=0$. Now, by Proposition 3.9(d) one gets $S_{r}\left(A_{j_{1}}, A_{\theta}\right)=0$. Taking $r=n-2$ and $\theta=j_{2}$ we get

$$
S_{n-2}\left(A_{j_{1}}, A_{j_{2}}\right)=\prod_{\substack{i=1 \\ i \neq j_{1}, j_{2}}}^{n} \lambda_{i}=0
$$

therefore, there exists another null principal curvature which we will denote by $\lambda_{j_{3}}=0$. Again, by Proposition 3.9(g), one gets $S_{r}\left(A_{j_{1}}, A_{j_{2}}, A_{\theta}\right)=0$. Taking $r=n-3$ and $\theta=j_{3}$ we get

$$
S_{n-3}\left(A_{j_{1}}, A_{j_{2}}, A_{j_{3}}\right)=\prod_{\substack{i=1 \\ i \neq j_{1}, j_{2}, j_{3}}}^{n} \lambda_{i}=0
$$


Therefore, there exists another null principal curvature which we will denote by $\lambda_{j_{4}}=0$.

Continuing in this fashion, we will show that there exist $n-k+1$ null principal curvatures.

Corollary 7.3. Let $x: \mathbf{M}^{n} \longrightarrow \overline{\mathbf{M}}^{n+1}, n \geq 3$ be a $k$-umbilical isometric immersion. If $H_{n}=0$ at one point, then $H_{j}=0$ at the same point, $\forall j \geq k$.

8. Two-umbilical Isometric Immersions. Here, we obtain the principal curvatures $\lambda_{1}, \ldots, \lambda_{n}$, of a two-umbilical isometric immersion in a Riemannian manifold $\overline{\mathbf{M}}^{n+1}$, in terms of $S_{1}$ and $S_{2}$, as roots of $\lambda_{i}^{2}-S_{1} \lambda_{i}+\frac{2}{n} S_{2}=0$ (cf. Remark 6.4).

THEOREM 8.1 (Determination of the principal curvatures of the two-umbilical isometric immersions). Let $x: \mathbf{M}^{n} \longrightarrow \overline{\mathbf{M}}^{n+1}(n \geq 3)$ be any two-umbilical isometric immersion.

(a) If its principal curvatures are distinct, then they are given by

$$
\lambda_{i_{1}}=\cdots=\lambda_{i_{r}}=((n-(r+1)) /(n-2 r)) S_{1}
$$

and

$$
\lambda_{i_{r+1}}=\cdots=\lambda_{i_{n}}=-((r-1) /(n-2 r)) S_{1},
$$

where $r \in\left\{0,1,2, \ldots,\left[\left[\frac{n}{2}\right]\right]^{4}\right\}$ or $r \in\left\{0,1,2, \ldots, \frac{n}{2}-1\right\}$, according to whether $n$ is odd or even, respectively.

(b) If its principal curvatures are equal, then $n$ is even and its principal curvatures are given by

$$
\lambda_{i_{1}}=\cdots=\lambda_{i \frac{n}{2}}=\sqrt{2 / n} \sqrt{-S_{2}}
$$

and

$$
\lambda_{i_{\frac{n}{2}+1}}=\cdots=\lambda_{i_{n}}=-\sqrt{2 / n} \sqrt{-S_{2}} .
$$

Proof. Let $\left\{e_{1}, \ldots, e_{n}\right\}$ be an orthonormal basis which diagonalizes $A$. Since $x$ is two-umbilical, by Remark 6.4 each $\lambda_{i}$ satisfies $\lambda_{i}^{2}-S_{1}(A) \lambda_{i}+\frac{2}{n} S_{2}(A)=0$; then each $\lambda_{i}$ has at most two distinct principal curvatures:

$$
\begin{aligned}
\lambda_{i_{1}}=\lambda_{i_{2}}=\cdots=\lambda_{i_{r}}= & \frac{S_{1}(A)+\sqrt{S_{1}(A)^{2}-\frac{8}{n} S_{2}(A)}}{2} ; \\
\lambda_{i_{r+1}}=\lambda_{i_{r+2}}=\cdots=\lambda_{i_{n}}= & \frac{S_{1}(A)-\sqrt{S_{1}(A)^{2}-\frac{8}{n} S_{2}(A)}}{2} .
\end{aligned}
$$

We then obtain

$$
S_{1}(A)=\frac{n S_{1}(A)+(2 r-n) \sqrt{S_{1}(A)^{2}-\frac{8}{n} S_{2}(A)}}{2} .
$$

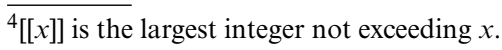


Suppose that $r \neq \frac{n}{2}$. Then $\sqrt{S_{1}(A)^{2}-\frac{8}{n} S_{2}}=\frac{(n-2) S_{1}(A)}{n-2 r}$. Now we have

(i) If $n \geq 2, S_{1} \geq 0$ and $r<\frac{n}{2}$, then the principal curvatures are given by

$$
\begin{aligned}
\lambda_{i_{1}} & =\cdots=\lambda_{i_{r}}=\left[\frac{n-(r+1)}{n-2 r}\right] S_{1}(A), \\
\lambda_{i_{r+1}} & =\cdots=\lambda_{i_{n}}=-\left(\frac{r-1}{n-2 r}\right) S_{1}(A) .
\end{aligned}
$$

(ii) If $n \geq 2, S_{1}(A) \leq 0$ and $r>\frac{n}{2}$, then the principal curvatures are given by

$$
\begin{gathered}
\lambda_{i_{1}}=\cdots=\lambda_{i_{r}}=\left[\frac{n-(r+1)}{n-2 r}\right]\left(-S_{1}(A)\right), \\
\lambda_{i_{r+1}}=\cdots=\lambda_{i_{n}}=-\left(\frac{r-1}{n-2 r}\right)\left(-S_{1}(A)\right) .
\end{gathered}
$$

Now suppose that $r=\frac{n}{2}$ and $n \geq 4$. Then $(n-2) S_{1}(A)=0$ and we obtain

$$
\begin{gathered}
\lambda_{i_{1}}=\cdots=\lambda_{i_{\frac{n}{2}}}=\sqrt{\frac{2}{n}} \sqrt{-S_{2}(A)}, \\
\lambda_{i_{\frac{n}{2}+1}}=\cdots=\lambda_{i_{n}}=-\sqrt{\frac{2}{n}} \sqrt{-S_{2}(A)} .
\end{gathered}
$$

COROllary 8.2. For any odd integer n, every minimal two-umbilical isometric immersion of $\mathbf{M}^{n}$ is one-totally geodesic.

From Remark 6.4 we can see that any two-umbilical immersion has no more that two principal curvatures, and Theorem 4.1 says that the maximum number of principal curvatures of any Einstein hypersurface immersed in a space form is two. Then arise a question: Is any two-umbilical manifold immersed in a space form an Einstein manifold? The answer is yes and it will be proved in the next theorem.

THEOREM 8.3 (A characterization of Einstein hypersurfaces). Let $\mathbf{M}^{n}$ be a connected Riemannian manifold and $x: \mathbf{M}^{n} \longrightarrow \overline{\mathbf{M}}^{n+1}(c), n \geq 3$, an isometric immersion. Then

$\mathbf{M}^{n}$ is Einstein if and only if $x$ is two-umbilical.

Moreover, in this case $\operatorname{Ric}(X, Y)=\left(c(n-1)+\frac{2 S_{2}}{n}\right)<X, Y>$, with $S_{2}$ constant.

Proof. Suppose $\mathbf{M}^{n}$ is Einstein. From Gauss equation

$$
\operatorname{Ric}(X, Y)-c(n-1)\langle X, Y\rangle=\left\langle A P_{1}(A) X, Y\right\rangle
$$

since $\mathbf{M}^{n}$ is Einstein, then $\operatorname{Ric}(X, Y)=\lambda\langle X, Y\rangle$, hence

$$
\left\langle A P_{1}(A) X, Y\right\rangle=(\lambda-c(n-1))\langle X, Y\rangle .
$$

Thus, $A P_{1}(A)=(\lambda-c(n-1)) I$ and the proof follows by using $(5.1)$. 
Now suppose $x$ is two-umbilical. By Gauss equation

$$
\operatorname{Ric}(X, Y)=\left(c(n-1)+\frac{2 S_{2}(A)}{n}\right)\langle X, Y\rangle
$$

therefore, $\mathbf{M}^{n}$ is an Einstein manifold. Moreover, because $\mathbf{M}^{n}$ is connected $(c(n-1)+$ $\frac{2 S_{2}(A)}{n}$ ) is constant, hence $S_{2}$ is constant.

REMARK 8.4. Let $x: \mathbf{M}^{n} \longrightarrow \overline{\mathbf{M}}^{n+1}, n \geq 2$ be an isometric immersion and $X, Y \in$ $T \mathbf{M}$. The Gauss equation and the definition of $\phi_{2}$ (Definition 5.6, for $k=2$ ) give that

$$
\operatorname{Ric}(X, Y)-\overline{\operatorname{Ric}}(X, Y)=\frac{2 S_{2}(A)}{n}\langle X, Y\rangle-\left\langle\phi_{2} X, Y\right\rangle
$$

From this we can see that the operator $\phi_{2}$ gives a measure of how much a manifold $\mathbf{M}$ immersed isometrically in a space form $\overline{\mathbf{M}}^{n+1}(c)$ fails to be an Einstein hypersurface.

9. Examples and Description of two-umbilical Hypersurfaces. An example of twototally geodesic immersion which is not one-totally geodesic is given by

$$
S^{1}(r) \times \mathbb{R}^{n-1} \subset \mathbb{R}^{n+1}
$$

EXAMPLE 9.1 (Clifford's hypersurfaces). Given $n_{1}, n_{2} \in \mathbb{N}$ and $r_{1}, r_{2}>0$. Consider

$$
\begin{aligned}
& S^{n_{1}}\left(r_{1}\right)=\left\{u \in \mathbb{R}^{n_{1}+1}:\|u\|=r_{1}\right\}, \\
& S^{n_{2}}\left(r_{2}\right)=\left\{u \in \mathbb{R}^{n_{2}+1}:\|u\|=r_{2}\right\}
\end{aligned}
$$

and

$$
S^{n_{1}}\left(r_{1}\right) \times S^{n_{2}}\left(r_{2}\right)=\left\{(u, v) \in \mathbb{R}^{n_{1}+n_{2}+2}: u \in S^{n_{1}}\left(r_{1}\right), v \in S^{n_{2}}\left(r_{2}\right)\right\}
$$

$S^{n_{1}}\left(r_{1}\right) \times S^{n_{2}}\left(r_{2}\right)$ is a hypersurface of $S^{n_{1}+n_{2}+1}(1) \subset \mathbb{R}^{n_{1}+n_{2}+2}$ with $r_{1}^{2}+r_{2}^{2}=1$ and it is called a Clifford's hypersurface.

Proposition 9.2. For any $n \in \mathbb{N}, 0<r<1$ and fixed $m \in\{1, \ldots, n-1\}$, the Clifford's hypersurface $S^{n-m}(r) \times S^{m}\left(\sqrt{1-r^{2}}\right) \longrightarrow S^{n+1}(1)$ has its principal curvatures given by

$$
\begin{gathered}
\lambda_{1}=\cdots=\lambda_{n-m}=\frac{\sqrt{1-r^{2}}}{r}, \\
\lambda_{n-m+1}=\cdots=\lambda_{n}=\frac{-r}{\sqrt{1-r^{2}}} .
\end{gathered}
$$

THEOREM 9.3 (Examples of two-umbilical hypersurfaces in $S^{n+1}(1)$ ). There exists a countably infinite family of two-umbilical hypersurfaces in the Euclidean sphere $S^{n+1}(1)$. More precisely: for any $n \geq 4$ and for every $m \in\{2, \ldots, n-2\}$, the Clifford's hypersurface

$S^{n-m}(r) \times S^{m}\left(\sqrt{1-r^{2}}\right) \hookrightarrow S^{n+1}(1) \quad$ is two-umbilical if and only if $\quad r=\sqrt{\frac{n-m-1}{n-2}}$. 
Furthermore, the norm of its second fundamental form $A$, the associated polynomial $S_{k}$ and its Ricci curvature are given by

$$
\begin{aligned}
S_{k} & =\left(\frac{m-1}{n-m-1}\right)^{\frac{k}{2}} \sum_{i=0}^{k}(-1)^{i}\left(\frac{n-m-1}{m-1}\right)^{i}\left(\begin{array}{c}
n-m \\
k-i
\end{array}\right)\left(\begin{array}{c}
m \\
i
\end{array}\right) ; \\
\|A\|^{2} & =\frac{n+m(n-4)(n-m)}{(n-m-1)(m-1)} ; \\
\operatorname{Ric}\left(e_{j}\right) & =n-2, \quad j=1,2, \ldots, n .
\end{aligned}
$$

Proof. By Proposition 5.9(2) we obtain

$$
\left\|\phi_{2}\right\|^{2}=\left(\frac{n-2}{n}\right) S_{2}\left(A_{\eta}\right)^{2}-S_{1}\left(A_{\eta}\right) S_{3}\left(A_{\eta}\right)-2 S_{4}\left(A_{\eta}\right)
$$

a short calculation shows that

$$
\left\|\phi_{2}\right\|^{2}=\frac{-m(m-n)}{2 n r^{4}\left(r^{2}-1\right)^{2}}\left((n-2) r^{2}-(n-m-1)\right) .
$$

The radius $r$ is obtained as a consequence of Remark 5.7 and we then conclude the proof.

REMARK 9.4. A straightforward computation shows that the Clifford's hypersurface with the above radius $r$ has

$$
\begin{aligned}
& S_{1}=\frac{2 m-n}{m-1} \sqrt{\frac{m-1}{n-m-1}}, \\
& S_{2}=\frac{-n}{2 !}, \\
& S_{3}=\frac{-(n-2)(2 m-n)}{3 !(m-1)} \sqrt{\frac{m-1}{n-m-1}} ;
\end{aligned}
$$

we then show that the hypersurfaces in Theorem 9.3 satisfy the condition $H_{3}=H_{1} H_{2}$ given in Theorem 7.1.

REMARK 9.5. If in Theorem 9.3 we make $n=2 \eta$ and $m=\eta+j$, we obtain a family of two-umbilical embedding:

$$
S^{\eta-j}(r) \times S^{\eta+j}\left(\sqrt{1-r^{2}}\right) \hookrightarrow S^{2 \eta+1}(1),
$$

where $r=\sqrt{\frac{\eta-j-1}{2 \eta-2}}, \eta \geq 2$ and $j \in\{0,1,2, \ldots, \eta-2\}$.

In this case, $S_{1}(A)=\frac{2 j}{\eta+j-1} \sqrt{\frac{\eta+j-1}{\eta-j-1}}$.

It is worth noting that $S_{1}(A)=0 \Longleftrightarrow j=0 \Longleftrightarrow r=\sqrt{\frac{1}{2}}$. Therefore, for every $\eta \in[2, \infty) \cap \mathbb{N}$ we have a minimal two-umbilical embedding

$$
S^{\eta}\left(\frac{1}{\sqrt{2}}\right) \times S^{\eta}\left(\frac{1}{\sqrt{2}}\right) \longrightarrow S^{2 \eta+1}(1)
$$


Thus, we have obtained a countably infinite family of minimal two-umbilical embeddings in the Euclidean sphere. Furthermore, for each $j=1,2, \ldots, \eta$

$$
\begin{aligned}
S_{2 j-1}(A) & =0 ; \\
S_{2 j}(A) & =(-1)^{j}\left(\begin{array}{l}
\eta \\
j
\end{array}\right) .
\end{aligned}
$$

THEOREM 9.6 (Description of the two-umbilical hypersurfaces in a space form). Let $\mathbf{M}^{n}$ be a two-umbilical hypersurface in $\overline{\mathbf{M}}^{n+1}(c), n>2$. Then

(a) $\mathbf{M}$ is two-totally geodesic or

(b) $\mathbf{M}$ is one-umbilical or

(c) if $c>0$, then $\mathbf{M}$ is locally a standard product embedding of

$$
S^{n-m}(r) \times S^{m}\left(\sqrt{1-r^{2}}\right) \hookrightarrow S^{n+1}(1),
$$

where $r=\sqrt{\frac{n-m-1}{n-2}}$. In particular, when the embedding is minimal we have

$$
S^{k}\left(\frac{1}{\sqrt{2}}\right) \times S^{k}\left(\frac{1}{\sqrt{2}}\right) \hookrightarrow S^{2 k+1}(1),
$$

where $n=2 k$;

(d) If $c<0$, then $\mathbf{M}$ is geodesic hyperspheres, horospheres, totally geodesic hyperplanes and their equidistant hypersurfaces, tubes around totally geodesic subspaces of dimension at least one (in another words, it is locally a standard product embedding given by $S^{k} \times \mathbb{\boxplus}^{n-k}$ );

(e) if $c=0$, then $\mathbf{M}$ is locally hyperspheres, hyperplanes or a standard product embedding given by $S^{k} \times \mathbb{R}^{n-k}$.

Proof. Theorem 8.1 on the determination of principal curvatures of two-umbilical hypersurfaces says that to know any two-umbilical hypersurface we need only to know $S_{1}(A)$ or $S_{2}(A)$. There are two cases to consider:

$$
S_{1}(A) S_{2}(A)=0 \quad \text { or } \quad S_{1}(A) S_{2}(A) \neq 0,
$$

where $A$ is the shape operator of $x$. We proceed with the study of each case.

(a) Suppose $S_{1}(A) S_{2}(A)=0$, with $S_{2}=0$ at one point. Since $S_{2}$ is constant (Theorem 8.3) then $S_{2} \equiv 0$. By Proposition 5.4 we have that $\mathbf{M}$ is two-totally geodesic. Now, we will show that $S_{1} \equiv 0$; in fact if Theorem $8.1(\mathrm{~b})$ is valid then we get $S_{1} \equiv 0$; if Theorem 8.1(a) is valid then we obtain $S_{2}=\frac{-n(r-1)[n-(r+1)]}{2(n-2 r)^{2}} S_{1}^{2}$, which implies either $S_{1} \equiv 0$ or $r=1$. In the first case we obtain one-totally geodesic hypersurfaces, in the other case we obtain two-totally geodesic hypersurfaces (only one mean curvature is non-null).

(b) Suppose $S_{1}(A) S_{2}(A) \neq 0$. Trivially any one-umbilical hypersurface (so it is twoumbilical and not minimal) satisfies this condition. Thus (b) is satisfied.

(c) Suppose $S_{1}(A) S_{2}(A) \neq 0$ and $\mathbf{M}$ is not one-umbilical. Theorem 8.1(b) cannot hold, because $\mathbf{M}$ would be minimal which is a contradiction. Since Theorem $8.1(\mathrm{a})$ is valid we can see that $S_{2}=\frac{-n(r-1)[n-(r+1)]}{2(n-2 r)^{2}} S_{1}^{2}$ and by Theorem 8.3 we get that $S_{1}(A)$ is constant. In this case the immersion is isoparametric. By using the Gauss-Codazzi equations, Cartan [5] proved that $\mathbf{M}$ is locally a standard product embedding of two spheres with appropriate radii. By Theorem 9.3 we 
get the radii. If $S_{2} \neq 0$ but $S_{1}=0$, we can see that only Theorem 8.1 (b) can hold, and so $S_{2}(A)<0$. If you suppose that $S_{1}=0$ and Theorem 8.1(a) is valid, then all its principal curvatures are equal to zero, and it follows that $S_{2}(A)=$ 0 , a contradiction. Hence, our hypersurface is minimal with two principal curvatures of multiplicity greater than two; the proof follows as a consequence of the corollary given in [9, Page 153] and Theorem 9.3.

(d) Using the Gauss-Codazzi equations, Cartan [5] proved that an isoparametric hypersurface $x: \mathbf{M}^{n} \rightarrow \mathbf{M}^{n+1}(-1)$ is either one-umbilical or has exactly two constant principal curvatures (see also [6]). This leads to the above classification.

As a consequence, all two-umbilical hypersurfaces in hyperbolic spaces are open parts of homogeneous hypersurfaces.

(e) By the same argument as given in (d). (See another proof in [10]).

\section{REFERENCES}

1. H. Alencar and M. do Carmo, Hypersurfaces with constant mean curvature in spheres, Proc. Am. Math. Soc. 120(4) (1994), 1223-1229.

2. A. C. Asperti and M. Dajczer, $N$-dimensional submanifolds of $\mathbb{R}^{N+1}$ and $S^{N+2}$, Illinois J. Math. 28(4) (1984), 621-645.

3. J. L. Barbosa and A. G. Colares, Stability of hypersurfaces with constant $r$-mean curvature, Ann. Global Anal. Geom. 15 (1997), 277-297.

4. A. Besse, Einstein Manifolds (Springer-Verlag, Berlin, 1987).

5. E. Cartan, Familles de Surfaces Isoparamétriques dans les Espaces à Courbure Constant, Annali di Mat. 17 (1938), 177-191.

6. M. Dajczer and M. do Carmo, Rotation hypersurfaces in spaces of constant curvature, Trans. Am. Math. Soc. 277(2) (1983), 685-709.

7. M. Dajczer and L. Florit, On Chen's basic equality, Illinois J. Math. 42(1) (1998) 97-106.

8. A. Fialkow, Hypersurfaces of a space of constant curvature, Ann. Math., 2nd Ser. 39(4) (1938), 762-785.

9. T. Otsuki, Minimal hypersurfaces in a Riemannian manifold of constant curvature, Am. J. Math. 92 (1970), 145-173.

10. H. Reckziegel, On the problem whether the image of a given differentiable map into a Riemannian manifold is contained in a submanifold with parallel second fundamental form, J. Reine Angew. Math. 325 (1981), 87-104.

11. R. Walter, Compact hypersurfaces with a constant higher mean curvature function, Math. Ann. 270 (1985), 125-145. 